\title{
Observations on Chiasmata in Mouse Diplotene Oocytes and Spermatocytes
}

\author{
Georgiana Jagiello and Jye-Siung Fang \\ Departments of Genetics and Development, \\ Obstetrics and Gynecology and the Center for Reproductive Sciences \\ of the International Institute for the Study of Human Reproduction, \\ College of Physicians and Surgeons of Columbia University, \\ 630 West 168th Street, New York, N.Y. 10032, U.S.A.
}

Accepted December 9, 1985

In 1932 Crew and Koller reported that crossing over values and chiasma frequencies were higher in female than male mouse germ cells. Implicit to this conclusion was the acceptance of the partial chiasmatype hypothesis (Janssens 1924, Darlington 1965), which equated chiasmata with crossing over and recombination due to crossing over. An analysis of a few diplotene chiasmata in oocytes and spermatocytes was presented by Crew and Koller as part of the evidence for a proposed sexual dimorphism and analysis of chiasmata frequencies in a large number of diplotene mouse oocytes and spermatocytes confirmed a greater total frequency in the female of the strain studied (Jagiello and Fang 1979). Several reports have been published which assessed chiasma frequencies at the later stages of diakinesis/metaphase I oocytes and spermatocytes of various mouse strains (Kysliková and Forejt 1972, Polani and Jagiello 1976, Speed 1977). Of particular interest was the finding of non-randomness of localization of chiasmata at this meiotic stage noted by Polani (1972).

In the present experiments, development of early diplotene chromomeric maps of mouse oocytes and spermatocytes has permitted exact bivalent identification and hence, exact localization of chiasmata. This methodology has been applied to the question of numerical and geographical relationships of chiasmata on each bivalent as a function of the sex of the germ cell.

\section{Materials and methods}

\section{Female}

Fifteen 40-60 day old cycling female random bred Swiss mice (CAMM) were mated to young males of proven fertility of the same strain. The pregnant females were sacrificed late on day $16(4 \mathrm{PM}$ ) or early on day $17(9 \mathrm{AM}$ ) of gestation (day of plug=0). Whole ovaries from thirty female fetuses were rapidly removed and placed in $0.05 \mathrm{M} \mathrm{KCl}$ at $38^{\circ}$ for $1 \mathrm{~h}$. They were then fixed with a freshly made solution of three parts absolute ethanol to one part glacial acetic acid, squashed and smeared in a drop of fix on clean slides. Slides were stained with $3 \%$ Giemsa (Fisher SO-G-28 diluted with distilled water and pH adjusted to 8.8 with $0.1 \mathrm{~N}$ ammonium hydroxide) for 5 to 10 minutes and then air-dried. Fifty intact well-spread early diplotene oocytes were chosen for sharpness of chromomere pattern and minimum bivalent overlapping (Fig. 1b) using a $10 \times$ and $100 \times$ Neofluar or Planapo lens of a Zeiss Photomicroscope II. These cells were photographed with the $100 \times$ Planapo lens using Kodak Technical Pan film 2415. Drawings of the complete bivalent complement of 15 of the 50 pre-photographed cells were then made using the $100 \times$ Planapo lens (Fig. 1c). Each bivalent was then identified in the original 50 photomicrographs using its relative length in the complement and its characteristic chromomere pattern as compared with the mid/late pachytene chromomere 
map of Jagiello and Fang (1980). Final identification of all bivalents (Fig. 2) was then developed by observing each bivalent of each complete cell with a $100 \times$ Planapo light and phase objective and simultaneously observing the drawings and photomicrographs.

Chiasmata were identified on each bivalent of fifteen cells of each sex by using a $100 \times$ Planapo lens (Fig. 1c). Their specific locations were assigned utilizing an updated version of the pachytene chromomere map nomenclature (Jagiello and Fang 1980) [Table 1 and Fig. 2].

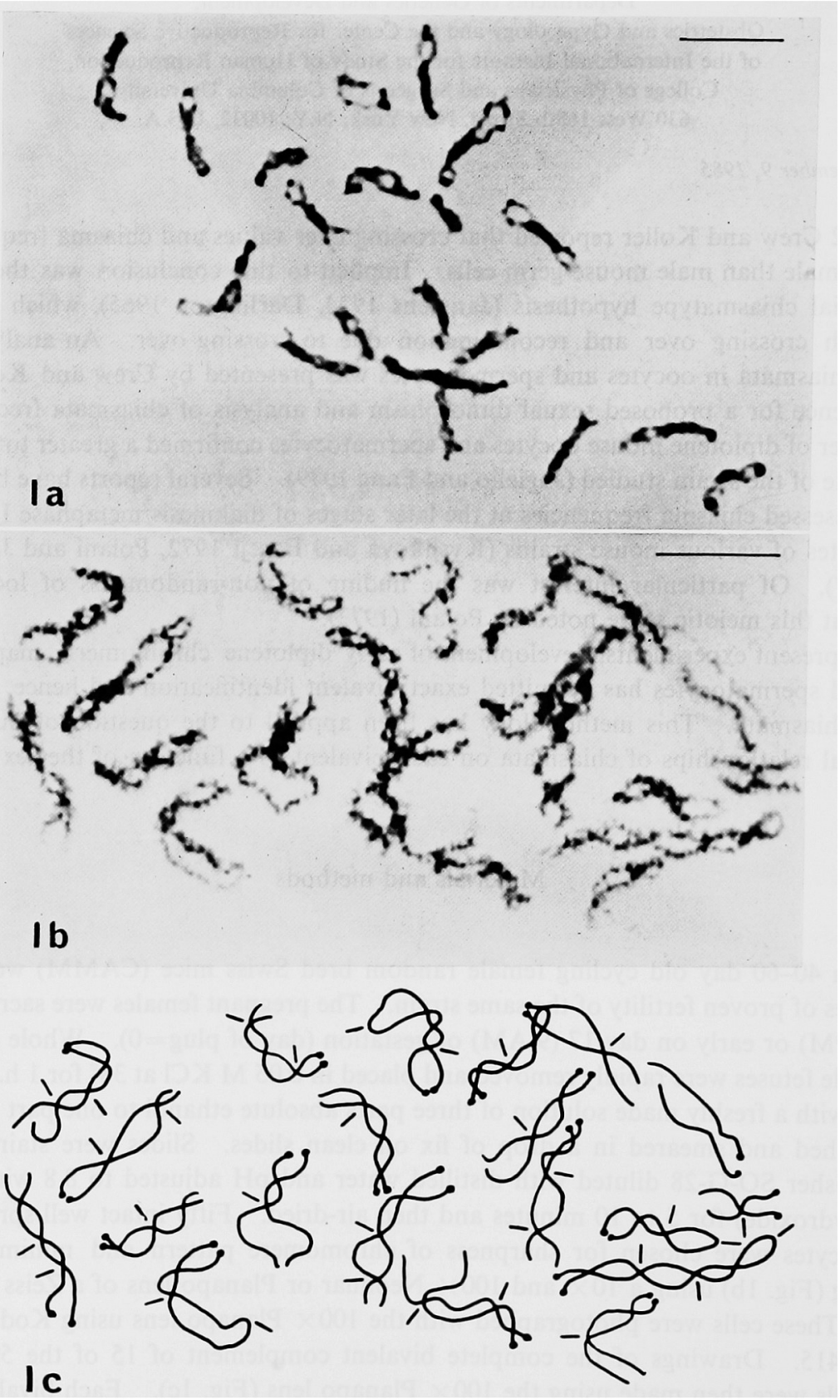

Figs. 1a-c. 1a, mouse: very early diplotene oocyte. $1300 \times .1 \mathrm{~b}$, mouse: early diplotene oocyte with maxium detectable chiasmata. $1500 \times$. The bars in $1 \mathrm{a}$ and $1 \mathrm{~b}$ represent $10 \mu \mathrm{m}$. 1c, diagram of bivalents in $1 \mathrm{~b}$; short lines indicate chiasmata positions. 
Great care was taken to correctly identify each chiasma microscopically and to avoid misinterpretation of artefacts by fractional microscopic focusing and tracing the length of each chromatid, as well as differentiation by staining intensity, since chiasma stains homogenously and regions of overlap stain more heavily. For purposes of comparison with other reports,

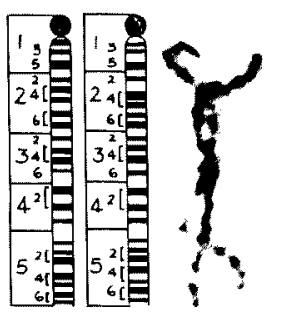

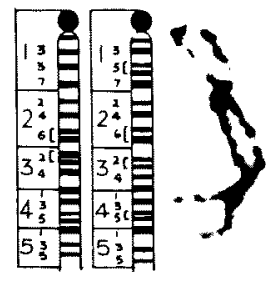

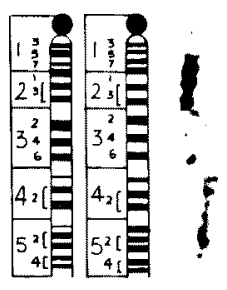

3

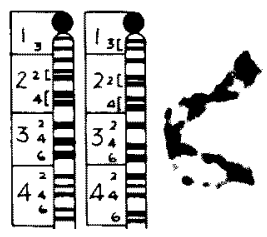

5

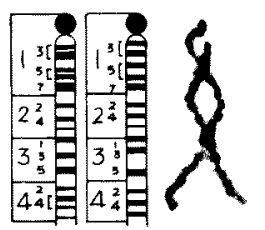

9

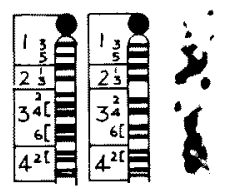

13

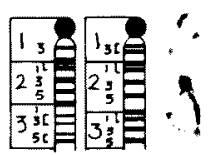

17

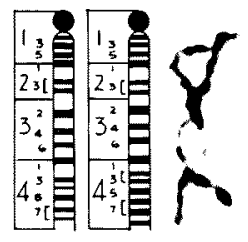

6

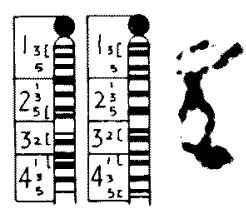

10

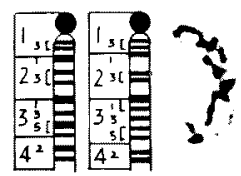

14

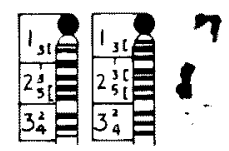

18

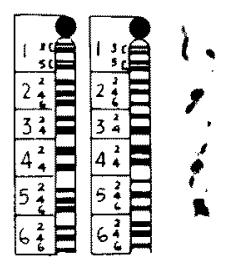

$x$

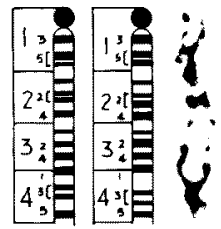

7

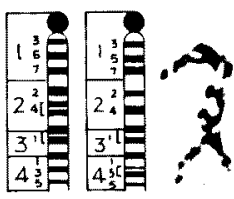

II

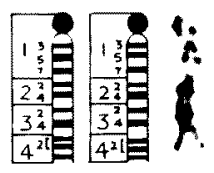

15

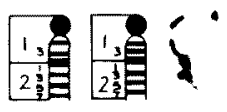

Fig. 2. Comparison of idiograms of (left) mid/late pachytene oocyte chromomeres, (center) early diplotene chromomeres and (right) representative diplotene bivalents. 
each bivalent was divided into proximal, central and distal thirds by measuring total length and dividing appropriately. A statistical analysis of the frequency of each chiasma location on each bivalent of oocytes compared with spermatocytes was done by chi square. This analysis was based on the probability that each chiasma had an equal chance of appearing in a germ cell of either sex.

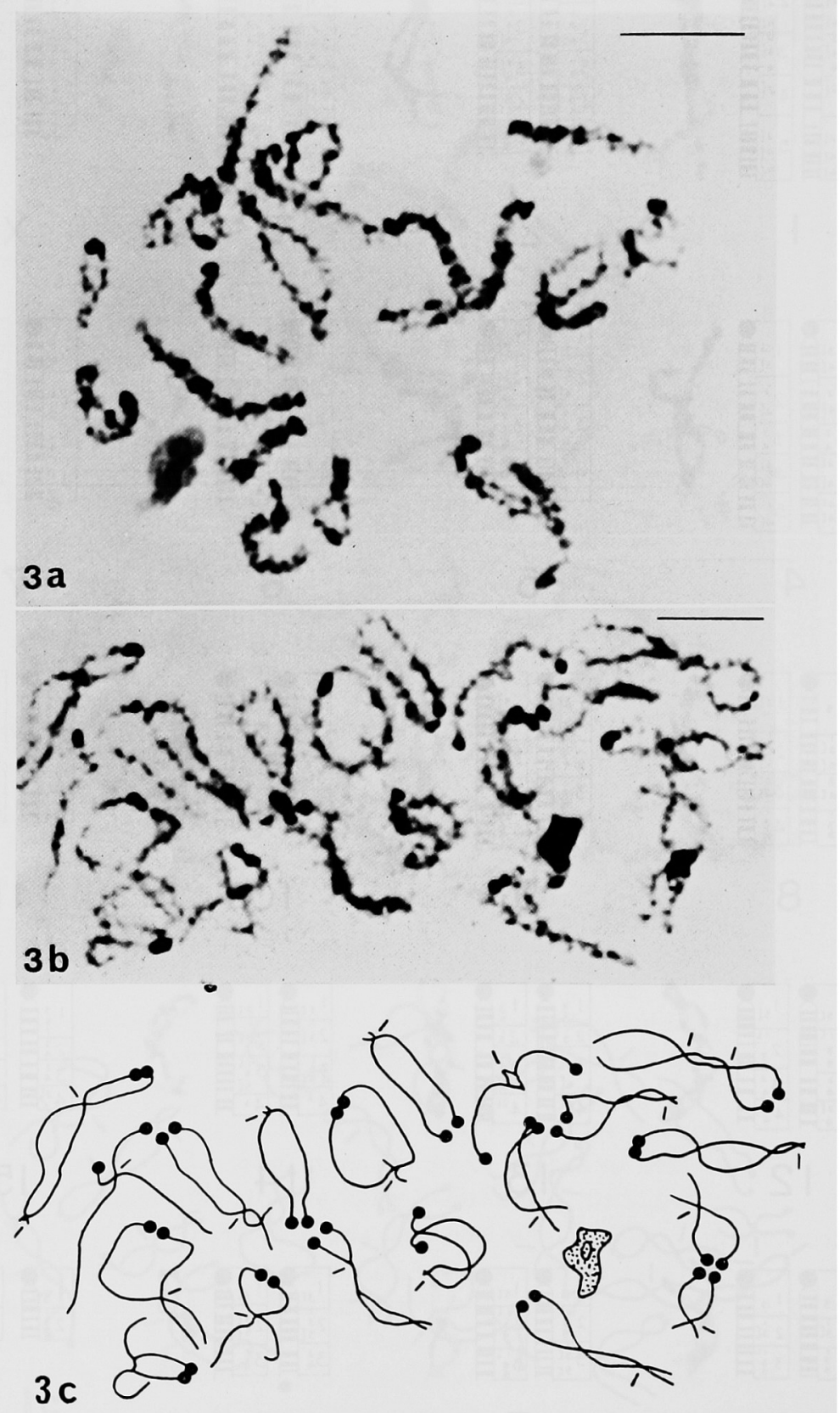

Figs. 3a-c. 3a, mouse: very early diplotene spermatocyte. $1500 \times$. 3b, mouse: early diplotene spermatocyte with maximum detectable chiasmata. $1300 \times$. The bars in $3 \mathrm{a}$ and $3 \mathrm{~b}$ represent $10 \mu \mathrm{m}$. 3c, diagram of bivalents in $3 \mathrm{~b}$; short lines indicate chiasmata positions. 


\section{Male}

Twelve male Swiss mice (CAMM) aged two-six months were studied. Both testes were rapidly removed after sacrificing by cervical dislocation and placed in Earle's balanced salt solution. Seminiferous tubules were dissected out and a loose bundle of tubules was placed in $0.07 \mathrm{M} \mathrm{KCl}$ at $38^{\circ} \mathrm{C}$ for $30 \mathrm{~min}$ to $1 \mathrm{hr}$. Tubules were fixed by the same method as the fetal ovaries. Very small segments (approximately $1 \mathrm{~mm}$ ) of tubule were selected and smeared on clean slides with a thin layer of fixative. These were then air-dried overnight. The procedures for staining, cell selection, chromomere mapping and bivalent identification, and

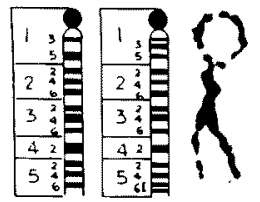

I

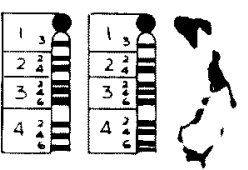

5

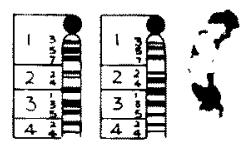

9

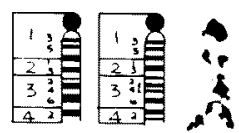

13

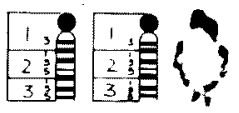

$4 \quad 17$

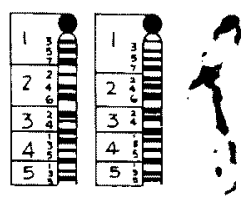

2

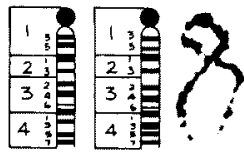

6

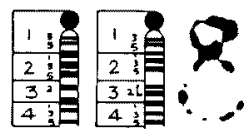

10

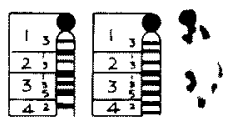

14

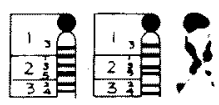

18

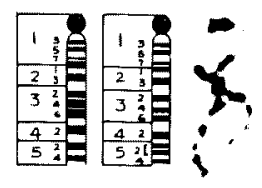

3

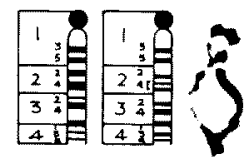

7

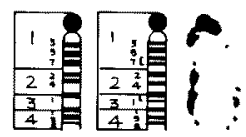

11

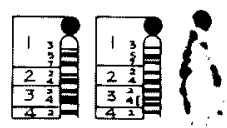

15

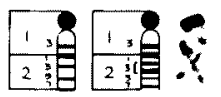

19

Fig. 4. Comparison of idiograms of (left) mid/late pachytene spermatocyte chromomeres, (center) early diplotene chromomeres and (right) representative diplotene bivalents.

chiasma localization (Figs. 3a, 3b, 3c) were carried out as in the experiments with the oocytes, except that an updated version of the pachytene chromomere map previously published for the male cells (Fang and Jagiello 1981) was used for assigning chiasmata to the individual bivalents (Table 1 and Fig. 4). 
288

Georgiana Jagiello and Jye-Siung Fang

Cytologia 52

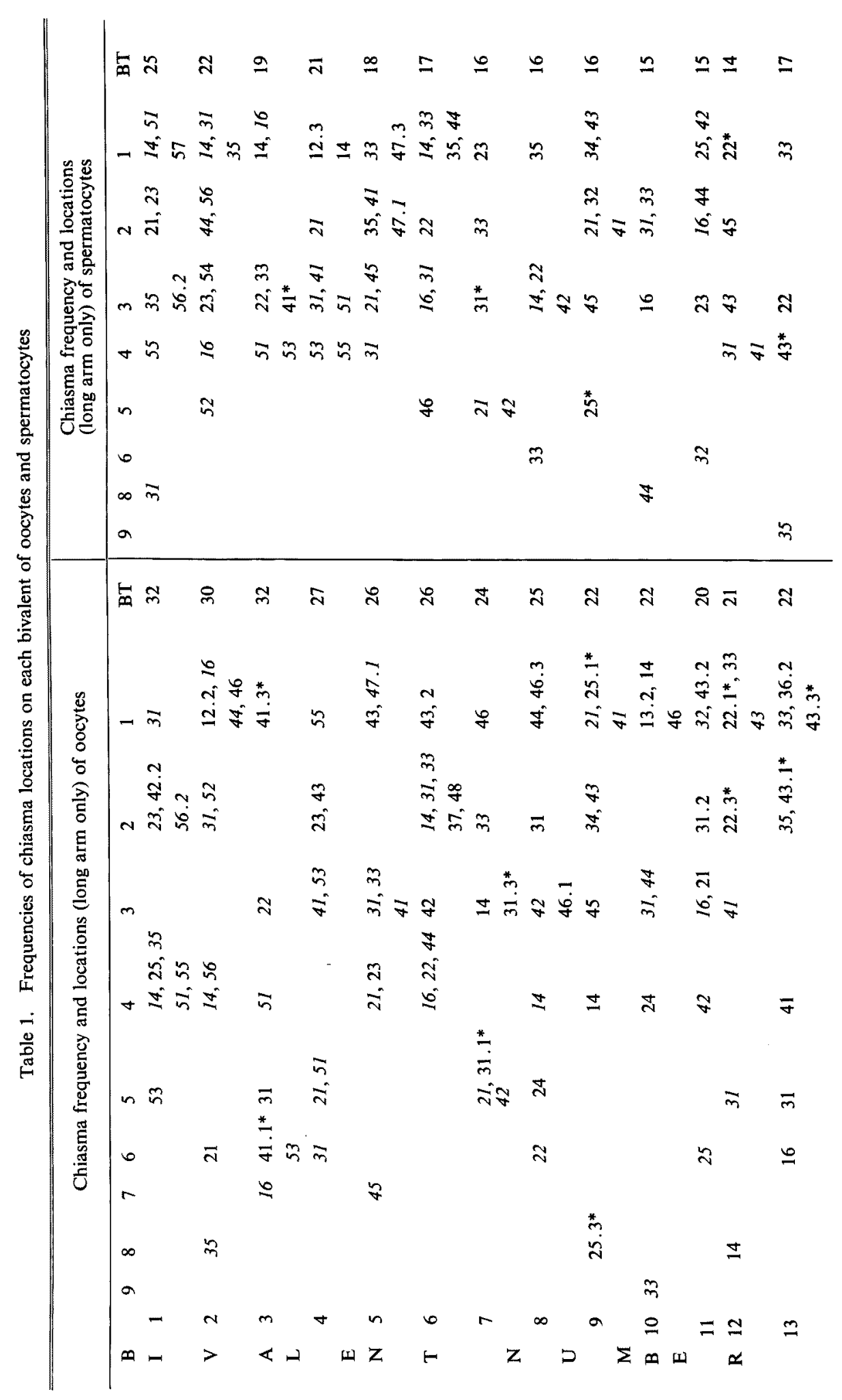




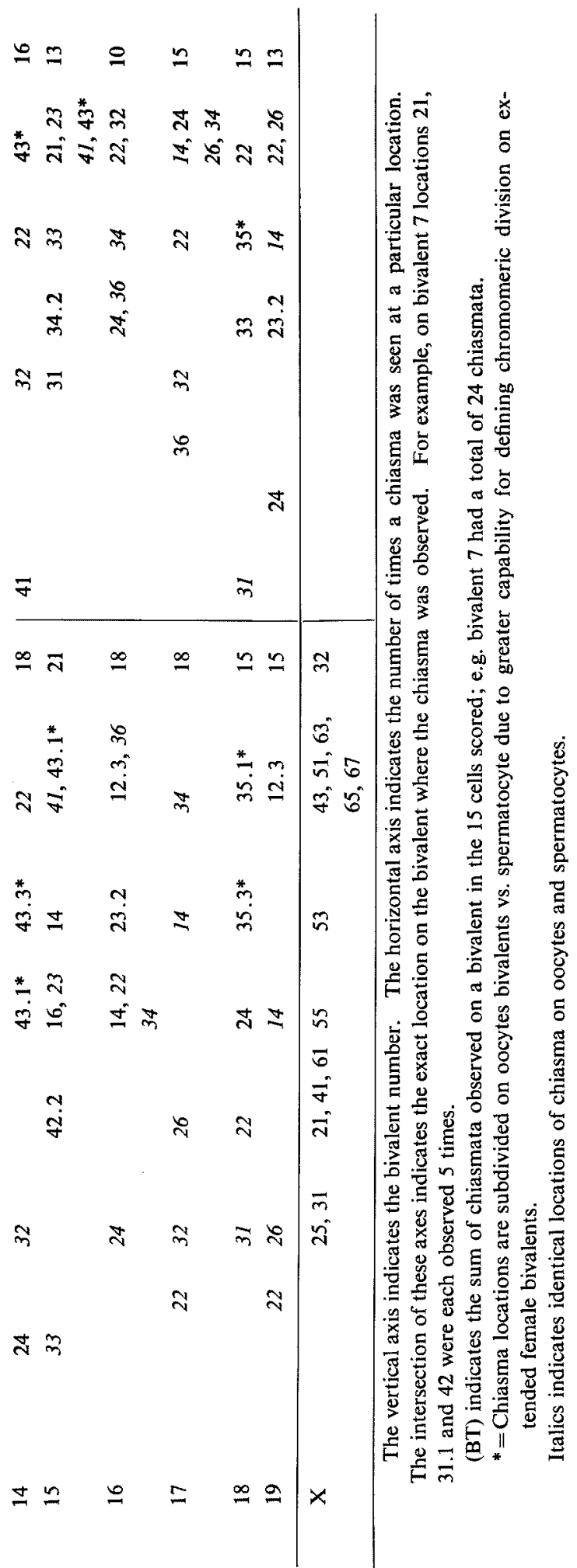




\section{Results}

As demonstrated in Figs. 1a and $3 a$, the transition from the pachytene to the diplotene stage proceeds asynchronously among the the bivalents. For both oocytes and spermatocytes, the chromomere maps of the early diplotene cells in which all bivalents have attained diplotene (Figs. 1b, 3b) were virtually identical with the previously observed mid/late pachytene maps (Figs. 2, 4) and each bivalent was thus easily identified by its chromomere pattern. The exact locations and frequencies of all observed chiasmata on analyzed oocytes and spermatocytes are tabulated in Table 1. All chiasmata appeared to be interchromomeric. Eighty-six out of $169(51 \%)$ chiasma locations were identical on oocytes and spermatocytes and are indicated

Table 2. Analysis of sexual dimorphism of chiasma locations and frequencies

\begin{tabular}{|c|c|c|c|}
\hline & & $\begin{array}{c}\text { Oocytes } \\
\text { Chiasma locations }\end{array}$ & $\begin{array}{c}\text { Spermatocytes } \\
\text { Chiasma locations }\end{array}$ \\
\hline B & 1 & 53,25 & 31 \\
\hline I & 2 & 35,21 & - \\
\hline $\mathrm{V}$ & 3 & 16,31 & - \\
\hline A & 4 & - & - \\
\hline $\mathbf{L}$ & 5 & 23 & - \\
\hline E & 6 & - & 46 \\
\hline $\mathbf{N}$ & 7 & - & - \\
\hline $\mathrm{T}$ & 8 & 24 & 33 \\
\hline & 9 & 14 & - \\
\hline $\mathbf{N}$ & & & \\
\hline & 10 & 33,24 & - \\
\hline $\mathrm{U}$ & & & \\
\hline & 11 & 25 & 32 \\
\hline M & & & \\
\hline $\mathrm{P}$ & 12 & 14 & - \\
\hline $\mathrm{B}$ & 13 & $16,31,41$ & 35 \\
\hline E & & & \\
\hline & 14 & 24 & 41 \\
\hline $\mathbf{R}$ & & & \\
\hline & 15 & $33,42.2$ & 31 \\
\hline & 16 & - & - \\
\hline & 17 & - & 36 \\
\hline & 18 & - & - \\
\hline & 19 & 22 & 24 \\
\hline
\end{tabular}

Chiasma locations (e.g. 53) listed by bivalent (e.g. bivalent 1$)$ were present significantly $(p<0.05)$ more often on oocyte bivalents if in the oocyte column, or more often on spermatocyte bivalents if listed under spermatocytes.

by underlining in Table 1. Fifty-two locations were unique to female cells and 31 to male cells. A total of 434 chiasmata were seen in 15 oocytes and 313 in 15 spermatocytes. The average number of chiasmata found per bivalent (sex chromosomes excluded) was 1.52 in oocytes and 1.10 in spermatocytes, in good agreement with the previous study of this strain which had demonstrated this sexual dimorphism (Jagiello and Fang 1979). There were more chiasmata observed in every female bivalent compared to male except bivalent 18 where equal numbers were detected in both sexes. The maximum difference of total chiasma formation between oocytes and spermatocytes (18 vs. 10) was noted for bivalent no. 16. Division of each bivalent length into thirds and using Fig. 2 and Table 1 yielded a pattern in the female bivalents of $24 \%$ proximal, $39 \%$ central and $37 \%$ distal chiasmata. In the male, using Fig. 4 and Table 
1 , it was found that the chiasmata were located $13 \%$ proximally, $33 \%$ centrally and $54 \%$ distally.

Examination of the frequencies of chiasma position summarized in Table 1 revealed nonrandomness. For example, on bivalent 1 of the oocytes, 5 of 15 cells had a chiasma at position 53 , and 4 had a chiasma at position $14,25,35,51$ and 55 , and positions $23,42.2$ and 56.2 were each found in two nuclei. In contrast, on bivalent 1 of the spermatocytes, 8 of 15 nuclei had a chiasma at position 31 , and 4 had a chiasma at position 55 , positions 35 and 56.2 were each found in three cells, and positions 21 and 23 were each found in two cells. Of the total of 434 chiasmata observed in the analyzed oocytes, $399(92 \%)$ consistently occurred in two or more cells. Of the 313 total chiasmata observed in spermatocytes, $275(88 \%)$ occurred in two or more cells. Comparisons of chiasma locations and frequencies between the sexes (Table 2) revealed no statistical difference between oocytes and spermatocytes on bivalents $4,7,16$ or 18 . On bivalents $2,3,5,9,10$ and 12 , one or two chiasma locations occurred more frequently in oocytes than spermatocytes; whereas on bivalents 6 and 17, one location was observed more often in the spermatocytes than the oocytes. On bivalents $8,11,14$ and 19 , a single different location occurred in either oocyte or spermatocyte. On bivalents 1,13 and 15 two more locations predominated in oocytes, but a single different location predominated in spermatocytes. The $\mathrm{X}$ bivalent of oocytes had a total of 32 chiasmata with a predominance of non-random locations at $21,25,31,41,55$ and 61 .

\section{Discussion}

Apparent persistence of major chromomeres from pachytene to diplotene has made possible the development of complete diplotene maps for mouse oocytes and spermatocytes. The capability for utilizing these maps in a meaningful way is presented by applying this method of diplotene bivalent identification to the question of the location and sexual dimorphism of chiasmata in this mouse strain.

The apparent interchromomeric location of all chiasmata does not support the suggestion of Lyon (1976) that mitotic chromosomal light $\mathrm{G}$ bands might be regions of low chiasma frequency. Dark $\mathrm{G}$ bands have previously been shown to correspond to pachytene chromomeres (Jagiello and Fang 1980, Fang and Jagiello 1981) as well as to diplotene chromomeres in the present study, hence were not considered to be chiasma sites.

The non-randomness of chiasmata indicated by the study of crossing over of Lyon (1976) was definitely observed in the present study. Consistency of individual sites of chiasmata was observed about equally in the oocytes $(92 \%)$ and the spermatocytes $(88 \%)$ and $71 \%$ of all chiasmata were similarly placed on both male and female autosomal bivalents. Of the remaining $29 \%$, approximately half were unique to the female and half to the male. In contrast to the analyses at diakinesis/MI of Polani (1972), it was observed in the present study of diplotene that there was a tendency to find more proximal chiasmata in the oocytes. Unlike Polani (1972) and Speed (1977), the present study revealed an equal distribution of centrally placed chiasmata in both diplotene oocytes and spermatocytes. However, findings in the present study of more distal chiasmata in the spermatocytes agreed with the data of Polani (1972) and Speed (1977).

The finding of sexual dimorphism of total chiasmata favoring the mouse female germ cell reported by Crew and Koller (1932), Polani (1972) and Jagiello and Fang (1979) was extended in the present study to individual autosomal bivalents. Every female bivalent had more chiasmata than the male, with the exception of bivalent 18 , but examination of individual locations, and their frequency of occurrence in each sex clearly indicated that the female used more of all sites more often than the male. These numerical findings and the localization data support 
the suggestion of Lyon (1976) that sex differences in recombination values favoring the female may be explained by chiasma locations.

The relevance of the locations of chiasmata to the questions of terminalization and preferential location of chiasma formation cannot be finally assessed from the present data base, but many more observations of the type presented here for this strain and of many inbred strains should be informative. Comparison of such data with currently available recombination values may also illuminate the question of gene clustering. The use of diplotene chromomere maps for bivalent identification extends many fold the capability for making such exact observations.

\section{Abstract}

Complete chromomere maps of Swiss mouse in early diplotene oocytes and spermatocytes have been developed. Individual bivalent identification has been used to assign all chiasmata in complete cells of each sex. All chiasmata appeared to be interchromomeric. More chiasmata were found on all oocyte bivalents than spermatocyte, except bivalent 18 where they were equal. There appeared to be non-random distribution of chiasmata in both sexes and 86 out of 169 chiasmata locations were identical on oocytes and spermatocytes. Differences of location between the present study of early diplotene cells and other reports of studies at the later meiotic stages of diakinesis/metaphase I were found.

\section{Acknowledgment}

This work was supported by the National Institutes of Child Health and Human Development Grant No. HD 10037.

\section{Literature cited}

Crew, F. A. E. and Koller, P. Ch. 1932. The sex incidence of chiasma frequency and genetical crossing over in the mouse. J. Genet. 26: 359-383.

Darlington, C. D. 1965 . Cytology. J. and A. Churchill, London, pp. 515.

Fang, J. S. and Jagiello, G. 1981. A pachytene map of the mouse spermatocyte. Chromosoma 82: 437-445.

Jagiello, G., Fang, J. S., Turchin, H. A., Lewis, S. E. and Gluecksohn-Walesch S. 1976. Cytological observations of deletions in pachytene stages of oogenesis and spermatogenesis in the mouse. Chromosoma 58: 377-386.

- and - 1979. Analyses of diplotene chiasmata frequencies in mouse oocytes and spermatocytes in relation to ageing and sexual dimorphism. Cytogenet. Cell Genet. 23: 53-60.

- and - 1980. A pachytene map of the mouse oocyte. Chromosoma 77: 113-121.

Janssens, F. A. 1924. La chiasmatypie dans les insectes. Cellule 34: 133-359.

Kysliková, K. and Forejt, J. 1972. Chiasma frequency in three inbred strains of mice. Folia Biologica 18: 216-218.

Lyon, M. F. 1976. Distribution of crossing over in mouse chromosomes. Genet. Res. 28: 291-299.

Polani, P. E. 1972. Centromere localization at meiosis and the position of chiasmata in the male and female mouse. Chromosoma 36: 343-374.

- and Jagiello, G. 1976. Chiasmata, meiotic univalents and age in relation to aneuploid imbalance in mice. Cytogenet. Cell Genet. 16: 505-529.

Speed, R. M. 1977. The effects of ageing on the meiotic chromosomes of male and female mice. Chromosoma 64: 241-254. 